INPLASY

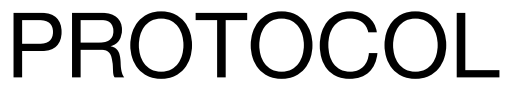

To cite: Webair et al. Health seeking behaviour among patients suffering from infertility in the Arab countries; a scoping review protocol. Inplasy protocol 202230034. doi:

10.37766/inplasy2022.3.0034

Received: 08 March 2022

Published: 08 March 2022

Corresponding author:

Hana Hasan Webair

hhwebair@gmail.com

Author Affiliation:

Hadhramout University, Universiti Sains Malaysia.

Support: No financial support.

Review Stage at time of this submission: Preliminary searches.

Conflicts of interest: None declared.

\section{Health seeking behaviour among patients suffering from infertility in the Arab countries; a scoping review protocol}

\author{
Webair, $\mathrm{HH}^{1}$; Ismail, TAT2; Ismail, $\mathrm{SB}^{3}$.
}

Review question / Objective: To identify how much and what is already known about health-seeking behavior (HSB) among the Arab patients who experienced infertility. Our purpose is to map and describe the studies that have been done and what they assessed concerning HSB among patients who experienced infertility. This includes the studies which address the factors affecting HSB. This review is conducted to display gaps in HSB literature and to inform a systematic review in the Arab countries.

Condition being studied: The review will study research articles which addressed the HSB among couples, men, or women suffering from infertility. We adopted the definition of HSB by Ward et al. (1997) which is the actions undertaken by the patients who perceive themselves as infertile for the purpose to conceive and get children (Ward, Mertens, \& Thomas, 1997). This could be any action ranged from neglect to seeking advanced infertility care. We will study the operational definition of HSB in each study, HSB model, rate of seeking medical care and type of care sought, other sources of help sought, and factors influencing HSB. In addition, we will describe how HSB was studied by defining the characteristics of the retrieved studies including design, setting, participants, and sample size, and infertility operational definition.

INPLASY registration number: This protocol was registered with the International Platform of Registered Systematic Review and Meta-Analysis Protocols (INPLASY) on 08 March 2022 and was last updated on 08 March 2022 (registration number INPLASY202230034).

\section{INTRODUCTION}

Review question / Objective: To identify how much and what is already known about health-seeking behavior (HSB) among the Arab patients who experienced infertility. Our purpose is to map and describe the studies that have been done 
and what they assessed concerning HSB among patients who experienced infertility. This includes the studies which address the factors affecting HSB. This review is conducted to display gaps in HSB literature and to inform a systematic review in the Arab countries.

Rationale: Infertility is a common problem worldwide and is associated with major psychosocial burden. The prevalence of infertility worldwide is around $10 \%$ but it is significantly higher in the Middle East and North Africa region (MENA) reached to 15\% (Colliers, 2021). MENA region includes all the Arab league countries. There are many effective solutions to manage infertility and help couples to get children and alleviate the stress of being childless. Surprisingly, only $56 \%$ of infertile patients are seeking medical care based on an international estimate of infertility treatment seeking (Boivin, Bunting, Collins, \& Nygren, 2007). Several studies had been conducted to explore the HSB related to infertility and the factors affecting it to help infertile couple to get benefit from the infertility services. There is a recent review included only developed countries as defined by the United Nations 2014 which did not show any country from Arab league (PassetWittig and Greil, 2021). Such review found the majority of studies were from US and therefore recommended multi-country studies to understand the process of HSB and help couples to overcome the obstacles and seek infertility care. To the best of our knowledge, HSB have not yet been conceptualized in the Arab countries. We aim to map the studies about HSB among infertile couples in Arab countries to identify the literature gaps and inform systematic review to synthesize the concept of HSB in the region.

Condition being studied: The review will study research articles which addressed the HSB among couples, men, or women suffering from infertility. We adopted the definition of HSB by Ward et al. (1997) which is the actions undertaken by the patients who perceive themselves as infertile for the purpose to conceive and get children (Ward, Mertens, \& Thomas,
1997). This could be any action ranged from neglect to seeking advanced infertility care. We will study the operational definition of HSB in each study, HSB model, rate of seeking medical care and type of care sought, other sources of help sought, and factors influencing HSB. In addition, we will describe how HSB was studied by defining the characteristics of the retrieved studies including design, setting, participants, and sample size, and infertility operational definition.

\section{METHODS}

Search strategy: Search strategy and information source: We will search MEDLINE (Pubmed), Google Scholar (the 200 first relevant references), review studies in the Cochrane Library (CENTRAL), and Prospero. Furthermore, the reference lists of all included articles will be searched to identify any additional, potentially eligible studies not captured by the electronic searches. We will search articles from Jan 2000- Feb 2020 Research articles published in English or Arabic only will be included. We will use the filter of date and language. Countries will not be added to search terms in order not to miss important studies because many titles and abstracts do not specify the study settings. The search terms used in Pubmed are; (( (health care seeking behaviour[MeSH Terms]) OR (help seeking[Text Word])) OR ("Health services use*"[Text Word])) AND ((infertility[MeSH Terms]) OR ("infertility care"[Text Word]) OR ("fertility care"[Text Word]) OR (reproductive techniques assisted[MeSH Terms])) The search terms will be tailored to the other electronic databases.

Participant or population: The review will include studies conducted on men, women, or couples suffering from infertility and desire to conceive regardless how the infertility diagnosis was made, and how old they are. We did not specify a definition for infertility but instead relied on the what the studies considered as infertile patients whether it was based on a medical diagnosis or patient perception and suffering of difficulty to conceive. That is 
because our review addresses the HSB which is triggered by the patient perception of the illness and not necessarily the medical diagnosis. Moreover, this review will not include diagnostic or therapeutic interventions which require a priori research diagnostic criteria to ensure reliability and generalizability of the results. We will exclude studies which was conducted on homosexual patients even if they suffered from difficulty to conceive because of the substantial differences in the reproductive solutions, psychology and social impact of infertility in case of homosexual versus heterosexual patients.

Intervention: No intervention.

\section{Comparator: No comparator.}

Study designs to be included: Research papers of all designs including review articles, interventional studies, crosssectional, case-control, and cohort designs will be included. Case series/reports, editorial reviews, letters of communications, and commentaries will be excluded. Articles with no full text available like conference abstracts will be excluded.

Eligibility criteria: Eligible studies are those which studied HSB of men, women, or couples suffering from infertility and desire to conceive regardless how the diagnosis of infertility was made, and how old they are. The outcome is the HSB which is defined here as any action taken by the patients who perceive themselves as infertile for the purpose to get children. Based on this definition; our study will include all forms of infertility treatment sought to solve the problem of childlessness wither medical or nonmedical. In addition, we will include articles which studied the factors affecting the HSB. We did not specify any interventions or control group for the eligibility. The participants should be from the Arab countries population to be included. We defined Arab countries as the $\mathbf{2 2}$ members of the Arab League (Comoros, Algeria, Bahrain, Djibouti, Egypt, Iraq, Jordan, Kuwait, Lebanon, Libya, Mauritania, Morocco, Oman, Palestine, Qatar, Saudi
Arabia, Somalia, Sudan, Syria, Tunisia, the United Arab Emirates and Yemen) (World Population Review, 2022). If the study involved participants from mixed geography, we will include the study and will focus on the results retrieved from Arab countries only. We will include the studies in Arabic or English language only as the Arabic is the spoken language in the Arab countries and the English is the most commonly used language in the academic publications.

Information sources: Mentioned above under search strategy.

Main outcome(s): The main outcome is the infertile patients HSB as defined above, and the factors affecting it.

Additional outcome(s): The additional outcome is the characteristics of the included studies to describe how HSB has been studied.

Data management: We prepared Excel sheet to chart the data, each variable will be in a separate column, and each study will be in a separate row. The sheet was pretested by the research team and modifications done accordingly. We will extract the following data: study characteristics including study design, setting, participants, and sample size, operational definition of infertility and HSB, what the infertile patients did to solve the problem of infertility?, HSB model if specified, rate of seeking medical care and type of medical care sought, other sources of help sought, and factors influencing HSB. The process of data management will be conducted by two authors. It will start after we finish the database search. We will export all the retrieved references to EndNote software version 9. The duplication will be removed. One author will screen the title and abstract of the retrieved articles. The second author will check $30 \%$ of the included and excluded articles. Agreement should be at least $\mathbf{8 0} \%$ among reviewers. If $80 \%$, the small percentage of disagreement will be solved by discussing the articles with the third author to reach consensus. Thereafter, 
both reviewers will independently review the full text of the included articles to confirm their eligibility. Then, the first author will extract data in the Excel sheet and the second author will review the extracted data aiming for at least $80 \%$ agreement.

Quality assessment / Risk of bias analysis: This review is conducted to display gaps in HSB literature and to inform a systematic review in the Arab countries. Therefore, this review will not assess the effectiveness of the findings, the risk of bias, or the certainty of the evidence (GRADE) at this stage. We will assess the quality and risk of bias if we proceed to conduct the systematic review but not with our current scoping review.

Strategy of data synthesis: We will follow the PRISMA extension of the scoping review method (Tricco et al., 2018). We will map the extracted data in tabular form. In addition, a narrative summary from the included studies will be reported to answer the research objectives.

Subgroup analysis: Not applicable.

Sensitivity analysis: Not applicable.

Language: Articles in Arabic or English will be included.

Country(ies) involved: Yemen; Malaysia.

Keywords: Health-seeking behavior; service utilization; infertility, assisted reproductive technologies; Middle East; North Africa; Arab.

Dissemination plans: The result of this review will be published in an open access peer reviewed journal.

Contributions of each author:

Author 1 - Hana Hasan Webair - Defining the title and objectives of the protocol. All authors participated in designing the review, and writing the protocol.

Email: hhwebair@gmail.com
Author 2 - Tengku Alina Tengku Ismail - All authors participated in designing the review, and writing the protocol.

Email: dralina@usm.my

Author 3 - Shaiful Bahari Ismail - All authors participated in designing the review, and writing the protocol.

Email: shaifulb@usm.my 